\title{
Patient Weight-bearing after Pelvic Fracture Surgery-A Systematic Review of the Literature: What is the Modern Evidence Base?
}

\author{
Mark Rickman ${ }^{1}$, Bjorn-Christian Link ${ }^{2}$, Lucian B Solomon ${ }^{3}$
}

\section{Abstract}

Background: Little attention in the literature appears to have been paid to the issue of postoperative weight-bearing protocols for different injury patterns after pelvic fracture surgery. The primary aim of this study is to review the currently available literature to define the level of available evidence used to inform surgical decisions on weight-bearing after pelvic fracture surgery. Secondary aims are to assess the published methods of fracture classification, surgical management, and assessment or reporting of patient outcomes.

Methods: A systematic review of the English language literature from 1990 to 2016 was undertaken. Eligible papers were all papers reporting minimum 6-month outcomes following surgery for pelvic fractures in adults. Exclusion criteria included pathological fractures or those resulting from penetrating injury, solely osteoporotic fractures, or series with less than 6 months of follow-up data.

Results: There is very little published scientific data to inform the treating surgeon on postoperative weight-bearing protocols after pelvic fracture surgery, with no randomized trials and only 1 paper out of 122 stating this as a primary aim. More than half of the papers published did not state what postoperative protocol was employed. There is no standardization of outcome measures, with less than $20 \%$ of papers using the most common validated outcome scoring system; in contrast, there is good agreement on the use of either the Tile (75\%) or Burgess and Young (20\%) classification.

Limitations: Due to the lack of published studies looking at the topic of postoperative weight-bearing after pelvic fractures, no specific recommendations are possible. As large numbers of papers were included, they were not individually assessed for bias.

Conclusion: A review of postoperative weight-bearing regimes reveals a nonexistent scientific evidence base from which to make recommendations, although a consensus strategy has been identified. Future research needs to be directed at this topic, as has already been the case in numerous other fracture areas, since the advantages of early mobility are potentially significant. The reported methodology for assessing and reporting patient outcomes after pelvic fracture surgery reveals no consistent standards, and the majority of papers use no specific outcome scoring system.

Keywords: Pelvic fracture, Pelvis, Rehabilitation, Weight-bearing.

Strategies in Trauma and Limb Reconstruction (2019): 10.5005/jp-journals-10080-1414
\end{abstract}

\section{INTRODUCTION}

Surgery for pelvic fractures has become more common over the last three decades, as a result of improvements in all aspects of care for the traumatically injured patient. For all but the most severe cases, there has been a steady improvement in mortality rates, ${ }^{1}$ and interest has increased in improving clinical outcomes for these patients. Modern fracture management is becoming more focused on techniques that facilitate early physiological rehabilitation, including early mobility and weight-bearing where possible. This is most commonly appreciated in the management of elderly trauma patients and, especially, the neck of femur fracture patients, but also applies to most other fracture regions, and has been one of the drivers for implant change and development.

Tradition dictates that the majority of patients following pelvic fracture surgery are kept nonweight-bearing, or, at least, minimal weight-bearing for several weeks, although protocols vary significantly from institution to institution. As a result, the primary aim of this paper is to interrogate the published literature in an attempt to identify the evidence base that exists to guide postoperative management, and, specifically, weight-bearing protocols, after pelvic fracture surgery. Secondary aims are to review the methods reported within the published literature to classify pelvic ring fractures and report clinical outcomes.

\begin{abstract}
${ }^{1-3}$ Centre for Orthopaedic and Trauma Research, Discipline of Orthopaedics and Trauma, University of Adelaide, Adelaide, Australia; Department of Orthopaedics and Trauma, Royal Adelaide Hospital, Adelaide, Australia

Corresponding Author: Mark Rickman, Centre for Orthopaedic and Trauma Research, Discipline of Orthopaedics and Trauma, University of Adelaide, Adelaide, Australia; Department of Orthopaedics and Trauma, Royal Adelaide Hospital, Port Road, Adelaide, Australia, Phone: +61 870741990, e-mail: mark.rickman@sa.gov.au

How to cite this article: Rickman M, Link B-C, Solomon LB. Patient Weight-bearing after Pelvic Fracture Surgery-A Systematic Review of the Literature: What is the Modern Evidence Base? Strategies Trauma Limb Reconstr 2019;14(1):45-52.
\end{abstract}

Source of support: Nil

Conflict of interest: None

\section{Methods}

A systematic review of the literature was carried out using the methods described in Preferred Reporting Items for Systematic Reviews and Meta-Analyses (PRISMA). ${ }^{2}$ The search terms used included "pelvic fracture," "pelvis fracture," "pelvic trauma," and "pelvic ring" for all databases, and results were limited to papers 
published between 1 January 1990 and 31 December 2016. Results were also limited to English language literature only, and human subjects. Pubmed was used as the main search engine for Medline, and further searches were performed using the Scopus and the Cochrane Central Register of Controlled Trials.

\section{Eligibility Criteria}

\section{Participants}

Skeletally mature patients suffering from an acute traumatic pelvic fracture, either alone or as a part of a multiple injury scenario.

\section{Intervention}

Operative management of the pelvic fracture.

\section{Comparison}

No comparison was required as all patients were included as one study group.

\section{Outcomes}

Results reported in any form, with a minimum follow-up period of 6 months from injury. Formal outcome scores were recorded, but studies were included where the only outcomes were descriptive rather than formal. Descriptions of postoperative weight-bearing regimes were recorded where given.

\section{Exclusion Criteria}

The major exclusions were abstracts and conference proceedings, review papers, and case reports. A series of pathological or specific osteoporotic fractures, as well as injuries secondary to penetrating trauma, were excluded to keep the pathology as uniform as possible. Reports with less than 6 months outcome data were excluded.

\section{Review Process}

The method of acquisition of the final list of included papers is shown in Figure 1. References were copied into the reference management software from all three searches, and duplicates were removed.

The abstracts were read in full, and papers included or excluded according to the above criteria. Any ambiguity at this stage resulted in the paper being sourced rather than rejected. The resulting list of papers was then sourced in full and read by two of the authors. Further papers were removed at this stage for a variety of reasons, such as registry reviews, technical papers, cadaveric studies, short follow-up not stated in the abstract, and theory-only papers with no clinical information. The final list of included papers was then analyzed independently by two of the authors and a table created collating data on patient numbers, injury types and classifications, methods of treatment, post-operative protocols, and assessment of outcomes. Any conflicts in inclusions were solved by discussion and, where necessary, arbitration with the third author.

\section{Results}

The initial search generated a collection of 7,590 articles. Limiting the selection to the English language resulted in 6,925 papers. Removing duplicate returns from the three databases searched, and applying exclusion criteria to the abstracts resulted in a group of 386 included abstracts. After all papers were sourced in full and read, further papers were removed according to the exclusion criteria leaving a final cohort of 122 papers $^{3-124}$ (see the method as described in Fig. 1).

The 122 papers covered a total number of 7,799 patients, with a mean of 64 and a median of 32 per paper (range 9-1,409). There were no prospective randomized trials identified, and only 13 papers ${ }^{11,12,14,37,66,77,104-106,120,121,123,125}$ included comparative cohorts, the remaining 109 papers all being case or cohort series.

Assessment of weight-bearing protocols after surgery revealed the following:

- There was only one paper ${ }^{7}$ where the stated main aim of the paper is to assess the effects of weight-bearing on patients' outcomes; this was restricted to vertically stable but rotationally unstable fracture types managed with external fixation.

- Sixty-three papers (52\%) did not state what amount of postoperative weight-bearing was allowed.

- Thirty-three papers (27\%) had patients touch- or nonweightbearing for a mean of 9 weeks. $4,5,9,11,12,17,25,28,29,32,36,37,39,40,43,52,54$, $58,59,61,74,77,81,85,89,95,111,113,114,121,126-128$

- Nineteen papers (15\%) had patients partially weight-bearing for a mean of 8 weeks. $16,24,45,57,61,63,66,75,76,82,118,119,123,124,129-133$

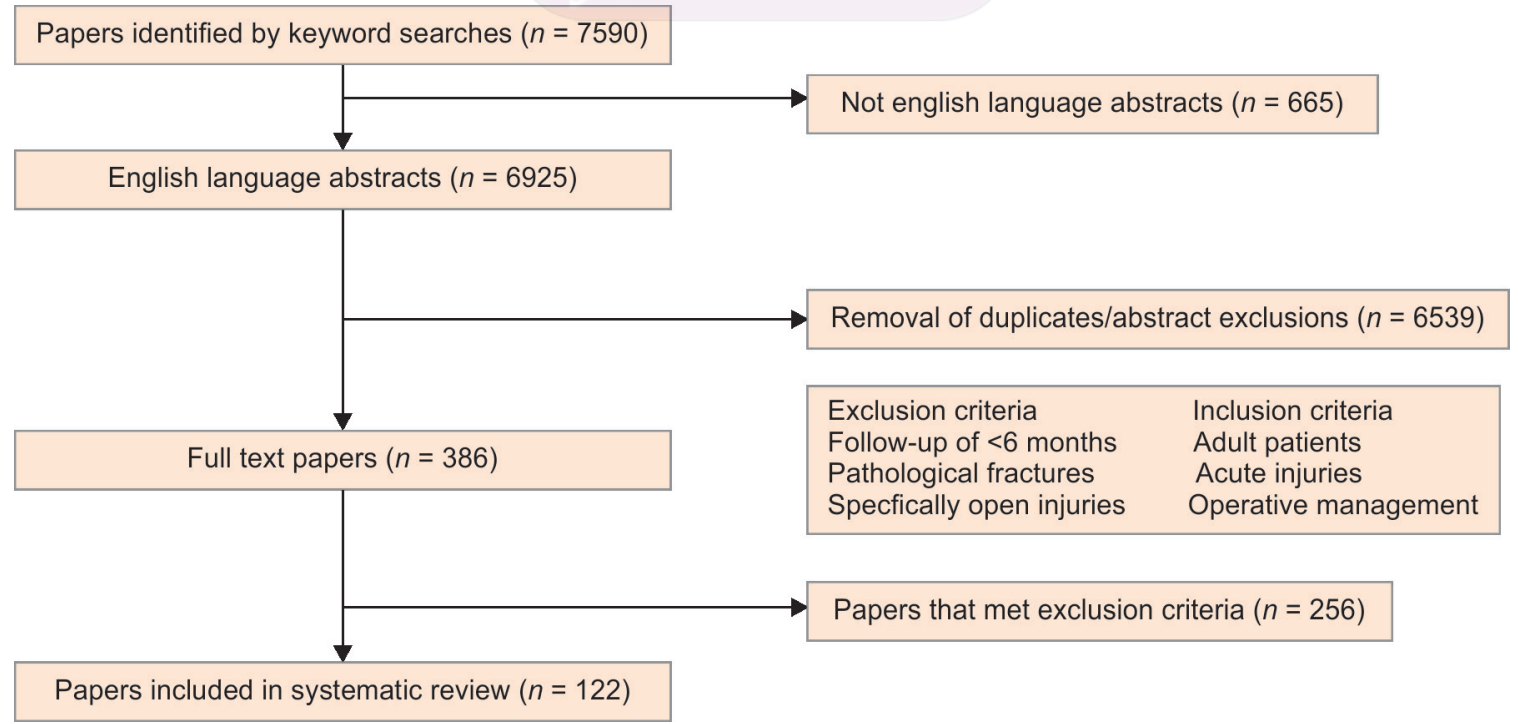

Fig. 1: Paper verification/exclusion process 
Table 1: Injury classifications reported

\begin{tabular}{lll}
\hline & Tile classification & \\
\hline Type A & Type B & Type C \\
\hline 765 patients (12.7\%) & $\begin{array}{l}\text { 1,917 patients (31.9\%) } \\
\text { Burgess and Young classification }\end{array}$ & 3,328 patients (55.4\%) \\
APC1-22 patients (1.6\%) & APC2-374 patients (26.9\%) & APC3-198 patients (14.2\%) \\
LC1-124 patients (8.9\%) & LC2-254 patients (18.2\%) & LC3-119 patients (8.5\%) \\
& Vert. shear-263 patients (18.9\%) & \\
& Combined-38 patients (2.7\%) & \\
\hline
\end{tabular}

- Seven papers (8.3\%) allowed full weight-bearing as tolerated in at least some patients, ${ }^{7,8,20,78,88-90}$ while two papers described enforced bedrest for 6 weeks.

Regarding injury classification, the most commonly used system was that of Tile ${ }^{134}$ (used in 92 papers), followed by the Burgess and Young classification ${ }^{135}$ (used in 24 papers). Eleven papers did not formally classify the injury type. The breakdown of injury types is given in Table 1.

The surgical management was varied across all types and is shown in Table 2. Table 3 shows the weight-bearing protocols employed for each Tile injury classification, and Table 4 shows the same weight-bearing data against the method of fixation.

Outcome measurements were highly varied across the papers with no standard method adopted. One hundred and three papers (85\%) used radiological methods as well as clinical to judge outcomes, 5 papers did not report specific outcome measures, and 65 papers gave specific nonvalidated clinical outcome measures such as pain, walking distance, and gait. Regarding validated scoring systems, the most commonly used system was the Majeed ${ }^{136}$ or the Lindahl $1^{137}$ version of it, but only 24 papers (19.7\%) used either method. Ten papers (8.2\%) reported SF-36 scores, and there were less than five papers using any other specific outcome scoring method.

There was no clear correlation between the fracture types treated and the weight-bearing protocols reported, or any apparent trend in the management over time. There was, however, a slight trend in the management of type $C$ fractures; of those papers reporting nonweight-

Table 2: Surgical management reported

\begin{tabular}{lll}
\hline Type of surgical procedure & $\begin{array}{l}\text { Number of } \\
\text { patients }\end{array}$ & $\begin{array}{l}\text { Percentage } \\
\text { of patients }\end{array}$ \\
\hline Ant ORIF and post percutaneous & 792 & 18.5 \\
Posterior percutaneous alone & 641 & 15 \\
Ant and post ORIF & 638 & 14.9 \\
Anterior ORIF alone & 548 & 12.8 \\
Anterior Exfix alone & 400 & 9.4 \\
Exfix plus posterior percutaneous & 393 & 9.2 \\
Posterior ORIF alone & 387 & 9.0 \\
Other & 235 & 5.5 \\
Ant and post percutaneous & 183 & 4.3 \\
Anterior percutaneous alone & 56 & 1.3 \\
\hline
\end{tabular}

Table 3: Cross table showing weight-bearing employed against Tile injury classification

\begin{tabular}{llll}
\hline Tile classification & Full weight & Partial weight & Non or touch weight \\
\hline A & 1 & 1 & 2 \\
B & 3 & 10 & 11 \\
C & 3 & 13 & 25 \\
\hline
\end{tabular}

Table 4: Cross table showing weight-bearing employed against type of surgical fixation-percentage of patients for each fixation method

\begin{tabular}{llcc}
\hline & \multicolumn{3}{c}{ Weight-bearing employed } \\
\cline { 2 - 4 } Fixation & Non or touch weight & Partial weight & Full weight \\
\hline 1 & 80 & 5 & 15 \\
2 & 59 & 41 & 0 \\
3 & 44 & 56 & 0 \\
4 & 71 & 27 & 2 \\
5 & 0 & 100 & 0 \\
6 & 7 & 89 & 4 \\
7 & 45 & 34 & 21 \\
8 & 78 & 19 & 3 \\
9 & 14 & 86 & 0 \\
\hline
\end{tabular}

Fixation method:

\begin{tabular}{ll}
\hline 1 & Anterior ORIF only \\
2 & Anterior ORIF plus percutaneous posterior \\
3 & Posterior ORIF only \\
4 & ORIF front and back \\
5 & Anterior percutaneous only \\
6 & Posterior percutaneous only \\
7 & Exfix alone \\
8 & Exfix plus posterior fixation \\
9 & Front and back percutaneous \\
\hline
\end{tabular}

bearing protocols, $86 \%$ included type $C$ fractures, the figure being $84 \%$ for partial weight-bearing protocols but only $33 \%$ for full weightbearing. The same figures for type B injuries were $56 \%$ for non- and full weight-bearing, and $68 \%$ for partial weight-bearing protocols.

Looking specifically at the subset of papers reporting only Tile type $C$ fractures (vertically unstable as well as rotationally unstable), there were 1,433 patients in 33 papers, with a mean of 44 per paper. Within this group, $22 \%$ underwent anterior open reduction and internal fixation (ORIF) plus percutaneous posterior fixation, 19\% had percutaneous posterior fixation only, and $35 \%$ had an open posterior procedure, with or without anterior fixation. No patients were managed with external fixation alone. Eleven papers did not state how patients were mobilized after surgery. Fourteen of the remaining papers reported non- or touch weight-bearing for a mean of 9.5 weeks. Six papers employed partial weight-bearing for the same time period, and two papers allowed full weight-bearing after fixation. ${ }^{20,78}$

\section{Discussion}

This systematic review of the available English literature publications on outcomes after surgically managed pelvic fractures 
was, primarily, to establish the level of evidence regarding weightbearing protocols after pelvic fracture surgery. The time period was defined from 1990 onwards with the aim of excluding historic papers, where management protocols generally included a high proportion of nonoperative management and long periods of bed rest.

It is clear that there is very little direct evidence to guide the treating surgeon on this subject, with no prospective trials and only 13 papers published with any sort of comparative cohort. In addition, surprisingly few papers used validated outcome scores that are comparable between methods and patient groups. Interpretation of this type of incomplete data with a view to informing surgical practice is inherently difficult.

There were no studies comparing the outcomes of protocols using different weight-bearing regimes. We identified only one paper where the stated aim was to investigate the effects of weightbearing after fixation, but this was in a limited subset of patients managed in a specific fashion. More concerning is the fact that over half of the papers identified by this review reported evidence on the outcome of pelvic fractures without stating what postoperative weight-bearing regime was employed. From the literature, however, there does appear to be some degree of consensus, in that, if patients are not allowed to bear full weight, then the mean time of restrictions is between 8 and 9 weeks, regardless of the fracture type or the level of restriction. In addition, there is a trend toward imposing more restrictions on type $C$ fractures rather than type $A$ or $\mathrm{B}$, although this is by no means universal.

There were two papers that allowed full weight-bearing as tolerated after fixation of solely type $C$ fractures. Duwelius et al. ${ }^{20}$ treated 16 type $C$ injuries in 13 patients using computerised tomography (CT)-guided ilio-sacral screws, with 9 patients also receiving anterior fixation. Schildhauer et al. ${ }^{78}$ reported 19 cases of spino-pelvic dissociation, all managed with lumbopelvic fixation and no anterior instrumentation. While neither paper reported formal outcome scores, both authors stated that all patients healed with no loss of fracture position, suggesting that with adequate stability full weight-bearing of even type $C$ injuries can be safe. Five other papers ${ }^{7,8,88-90}$ reported results after allowing full weightbearing in at least some cases. The only one to use specific outcome measurements (Majeed) was Tan et al., ${ }^{88}$ who reported on the use of lumbopelvic fixation for spinopelvic instability. Their results in just nine patients were five excellent or good outcomes, three fair, and one poor. The remaining four papers all managed type $B$ injuries with either external fixation or internal fixation, and all report predominantly good outcomes judged by various clinical indicators. No complications are reported in any of these seven papers that were attributed to the use of early weight-bearing by any of the authors.

Early weight-bearing after pelvic fracture surgery would have a number of possible benefits. Restricted weight-bearing and immobility are associated with a number of deleterious effects, most notably bone loss, muscle loss, and joint stiffness; mitigation against these problems should be beneficial to overall patient outcomes. In patients with multiple injuries, non- or touch weight-bearing is often impossible (e.g., due to upper limb injuries or contralateral lower limb injuries), leaving patients with pelvic fractures commonly restricted to bed or chair mobility for several weeks. Early weight-bearing may allow some of these patients to recover mobility earlier. In all patients (whether multiply injured or isolated pelvic fractures), earlier weight-bearing should lead to earlier discharge from hospital (as well as return to social and occupational activities), with concomitant financial and social benefits. We would postulate additional beneficial effects on venous thrombosis rates are associated, together with probable psychological benefits, with the earlier return of function in this high-risk group of patients.

There may be a number of reasons why the effects of early weight-bearing after pelvic fracture fixation have not been investigated, thus, far. There is an inherent fear that allowing patients to load fracture fixation may lead to loss of reduction and a poorer outcome; while this fear is entirely understandable, there appears to be little data to support it. Early weight-bearing has been investigated in other fracture regions, ${ }^{138-140}$ and in addition is known to be beneficial for long bone fracture healing. ${ }^{141}$ It may at first seem "unethical" to randomize patients between groups with differing weight-bearing regimes if the risk of one group is a failure of fixation; however, a well-constructed trial with informed consent as well as appropriate safeguards should negate this. It is also difficult to assess the exact level of weight-bearing activity patients have been doing, as patients' perceptions and reports of activity levels rarely match the reality. ${ }^{142-147}$ However, the drive for early weight-bearing after fracture fixation in other anatomic areas has led to improved fixation techniques and implants and, perhaps, is the path pelvic fracture fixation should follow.

One of the main issues with investigating weight-bearing after pelvic fracture fixation is our ability to measure fracture reduction and displacement in the first place. Plain X-rays have been shown to be inaccurate to measure fracture displacement of less than $5 \mathrm{~mm} .{ }^{148-150}$ Given the complexity of the pelvis, which makes a radiographic assessment of displacement more challenging than for other fractures, nonanatomic reduction and malunion have been defined as being in the order of at least a centimeter of displacement. ${ }^{151-154}$ Repeat CT scans are more accurate but involve high levels of radiation, while MRI scans are difficult to interpret after surgical fixation. In addition, to assess displacement early in the healing phase, imaging should be performed ideally with some degree of weight-bearing. One possible answer to this may be the use of radiostereometric analysis (RSA) technology. This has been shown to be accurate to the order of less than 0.01 $\mathrm{mm}$ in joint replacement surgery, ${ }^{155-157}$ and has been used at our institution to monitor tibial plateau fractures. ${ }^{158,159}$ With pelvic fractures, there may be some issues regarding the placement of the marker balls, especially if the surgery is percutaneous, but for most open procedures, it is a possible option and warrants further investigation. It is further limited though, in that few centers have the technology available to perform RSA analysis.

This paper does have limitations. We limited the papers to English language only and it is possible that the research question posed has been addressed in a different language. The papers selected were not assessed in any form for the quality of research or presence of bias. It was felt that with such large numbers of papers, this would be prohibitively time-consuming and would be unlikely to affect the outcomes of the review given the nature of the research question being asked. Despite a large number of papers included, there were no randomized studies. With the best available evidence today, it is not possible to make any firm recommendations. In addition, most published series do not specify the extent of other injuries sustained by patients which, in turn, has significant ramifications for postoperative weight-bearing. Given that the majority of long bone shaft fractures can be mobilized early after surgery, bilateral injuries or lower limb periarticular 
fractures are ones that should impact on weight-bearing regimes after a pelvic fracture. While this figure is impossible to obtain from the literature, at the authors' institutions, it would affect less than $25 \%$ of cases.

In conclusion, within the current literature, there is very little comparative data to inform the treating surgeon on the issue of weight-bearing after pelvic fracture surgery. There appears a current consensus for type $C$ fractures to be touch to partial weightbearing for 8-9 weeks, and for type B injuries partial weight-bearing for the same period of time; it is possible, however, for all pelvic fracture types to be managed with full weight-bearing as tolerated, given adequate stability. Injury classification within the literature appears to be relatively well standardized, but few papers report the use of validated outcome scores making useful interpretation of the data difficult.

\section{Ethical Approval}

This article does not contain any studies with human participants performed by any of the authors.

\section{References}

1. Pohlemann T, Stengel $D$, et al. Survival trends and predictors of mortality in severe pelvic trauma: estimates from the German Pelvic Trauma Registry Initiative. Injury 2011;42:997-1002. DOI: 10.1016/ j.injury.2011.03.053.

2. Liberati A, Altman DG, et al. The PRISMA statement for reporting systematic reviews and meta-analyses of studies that evaluate healthcare interventions: explanation and elaboration. BMJ 2009;339:b2700.

3. Adams MR, Scolaro JA, et al. The pubic midline exposure for symphyseal open reduction and plate fixation. J Orthop Trauma 2014;15:195-199. DOI: 10.1007/s10195-014-0296-9.

4. Aggarwal S, Bali K, et al. Management outcomes in pubic diastasis: our experience with 19 patients. J Orthop Surg Res 2011;6:21. DOI: 10.1186/1749-799X-6-21.

5. Albert MJ, Miller ME, et al. Posterior pelvic fixation using a transiliac $4.5 \mathrm{~mm}$ reconstruction plate: a clinical and biomechanical study. J Orthop Trauma 1993;7:226-232. DOI: 10.1097/00005131-199306000-00005.

6. Barei DP, Shafer BL, et al. The impact of open reduction internal fixation on acute pain management in unstable pelvic ring injuries. J Trauma 2010;68:949-953.

7. Bellabarba C, Ricci WM, et al. Distraction external fixation in lateral compression pelvic fractures. J Orthop Trauma 2000;14:475-482. DOI: 10.1097/00005131-200009000-00003.

8. Bellabarba C, Stewart JD, et al. Midline sagittal sacral fractures in anterior-posterior compression pelvic ring injuries. J Orthop Trauma 2003;17:32-37. DOI: 10.1097/00005131-200301000-00005.

9. Blake-Toker AM, Hawkins L, et al. CT guided percutaneous fixation of sacroiliac fractures in trauma patients. J Trauma 2001;51:1117-1121. DOI: 10.1097/00005373-200112000-00017.

10. Bungaro $P$, Rollo $G$, et al. Internal fixation in unstable fractures of the pelvis. Preliminary notes. Chir Organi Mov 1995;80:287-292.

11. Chen HW, Liu GD, et al. Treatment of unstable posterior pelvic ring fracture with percutaneous reconstruction plate and percutaneous sacroiliac screws: a comparative study. J Orthop Sci 2012;17:580-587. DOI: 10.1007/s00776-012-0257-1.

12. Chen $\mathrm{PH}, \mathrm{Hsu} \mathrm{WH}$, et al. Outcome analysis of unstable posterior ring injury of the pelvis: comparison between percutaneous iliosacral screw fixation and conservative treatment. Biomedical journal 2013;36:289-294. DOI: 10.4103/2319-4170.112757.

13. Cole JD, Blum DA, et al. Outcome after fixation of unstable posterior pelvic ring injuries. Clin Orthop Relat Res 1996;160-179. DOI: 10.1097/00003086-199608000-00020.
14. Cole PA, Gauger EM, et al. Anterior pelvic external fixator vs subcutaneous internal fixator in the treatment of anterior ring pelvic fractures. J Orthop Trauma 2012;26:269-277. DOI: 10.1097/ BOT.0b013e3182410577.

15. Dalbayrak S, Yaman O, et al. Surgical treatment in sacral fractures and traumatic spinopelvic instabilities. Turk Neurosurg. 2014;24:498-505.

16. Dienstknecht $T$, Berner A, et al. A minimally invasive stabilizing system for dorsal pelvic ring injuries. Clin Orthop Relat Res 2011;469: 3209-3217. DOI: 10.1007/s11999-011-1922-y.

17. Dolati $B$, Larndorfer $R$, et al. Stabilization of the posterior pelvic ring with a slide-insertion plate. Oper Orthop Traumatol 2007;19:16-31. DOI: 10.1007/s00064-007-1193-7.

18. Dong J, Hao W, et al. Management and outcome of pelvic fractures in elderly patients: a retrospective study of 40 cases. Chin Med J 2014;127:2802-2807.

19. Dujardin $\mathrm{FH}$, Hossenbaccus $\mathrm{M}$, et al. Long-term functional prognosis of posterior injuries in high-energy pelvic disruption. J Orthop Trauma 1998;12:145-150. DOI: 10.1097/00005131-199803000-00001; discussion 50-1.

20. Duwelius PJ, Van Allen M, et al. Computed tomography-guided fixation of unstable posterior pelvic ring disruptions. J Orthop Trauma 1992;6:420-426. DOI: 10.1097/00005131-199212000-00005.

21. Galois $L$, Pfeffer $F$, et al. The value of external fixation for unstable pelvic ring injuries. Acta Orthop Belg 2003;69:321-327.

22. Gansslen A, Hildebrand F, et al. Supraacetabular external fixation for pain control in geriatric type B pelvic injuries. Acta Chir Orthop Traumatol Cech 2013;80:101-105.

23. Gansslen A, Hufner T, et al. Percutaneous iliosacral screw fixation of unstable pelvic injuries by conventional fluoroscopy. Oper Orthop Traumatol 2006;18:225-244. DOI: 10.1007/s00064-006-1173-3.

24. Gansslen A, Krettek C. Retrograde transpubic screw fixation of transpubic instabilities. Oper Orthop Traumatol 2006;18:330-340. DOI: 10.1007/s00064-006-1181-3.

25. Gardner MJ, Mehta S, et al. Anterior pelvic reduction and fixation using a subcutaneous internal fixator. J Orthop Trauma 2012;26: 314-321. DOI: 10.1097/BOT.0b013e318220bb22.

26. Giannoudis PV, Chalidis BE, et al. Internal fixation of traumatic diastasis of pubic symphysis: is plate removal essential? Arch Orthop Trauma Surg 2008;128:325-331. DOI: 10.1007/s00402-007-0429-1.

27. Gras F, Marintschev I, et al. 2D-fluoroscopic navigated percutaneous screw fixation of pelvic ring injuries - a case series. BMC musculoskeletal disorders 2010;11:153. DOI: 10.1186/1471-2474-11-153.

28. Griffin DR, Starr AJ, et al. Vertically unstable pelvic fractures fixed with percutaneous iliosacral screws: does posterior injury pattern predict fixation failure? J Orthop Trauma 2006;20:S30-S36; discussion S6.

29. Gruen GS, Leit ME, et al. Functional outcome of patients with unstable pelvic ring fractures stabilized with open reduction and internal fixation. J Trauma 1995;39:838-844. DOI: 10.1097/00005373199511000-00006; discussion 44-5.

30. Gurevitz S, Bender B, et al. The role of pelvic fractures in the course of treatment and outcome of trauma patients. Isr Med Assoc J 2005;7:623-626.

31. Hamad A, Pavlou G, et al. Management of pubic symphysis diastasis with locking plates: a report of 11 cases. Injury 2013;44:947-951. DOI: 10.1016/j.injury.2012.12.018.

32. Hao $T$, Changwei $Y$, et al. Treatment of posterior pelvic ring injuries with minimally invasive percutaneous plate osteosynthesis. Int Orthop 2009;33:1435-1439. DOI: 10.1007/s00264-009-0756-7.

33. Hirvensalo $E$, Lindahl J, et al. A new approach to the internal fixation of unstable pelvic fractures. Clin Orthop Relat Res 1993;28-32. DOI: 10.1097/00003086-199312000-00007.

34. Hirvensalo E, Lindahl J, et al. Modified and new approaches for pelvic and acetabular surgery. Injury 2007;38:431-441. DOI: 10.1016/ j.injury.2007.01.020.

35. Hsu JR, Bear RR, et al. Open reduction internal fixation of displaced sacral fractures: technique and results. Orthopedics 2010;33:730. DOI: 10.3928/01477447-20100826-07. 
36. $\mathrm{Hu} \mathrm{SB}, \mathrm{Xu} \mathrm{H}$, et al. External fixation in early treatment of unstable pelvic fractures. Chin Med J 2012;125:1420-1424.

37. Hupel TM, McKee MD, et al. Primary external fixation of rotationally unstable pelvic fractures in obese patients. J Trauma 1998;45:111-115. DOI: 10.1097/00005373-199807000-00024.

38. Jacob AL, Messmer $P$, et al. Posterior pelvic ring fractures: closed reduction and percutaneous CT-guided sacroiliac screw fixation. Cardiovasc Intervent Radiol 1997;20:285-294. DOI: 10.1007/ s002709900153.

39. Jones $C B$, Sietsema DL, et al. Can lumbopelvic fixation salvage unstable complex sacral fractures? Clin Orthop Relat Res 2012;470:2132-2141. DOI: 10.1007/s11999-012-2273-z.

40. Kabak S, Halici M, et al. Functional outcome of open reduction and internal fixation for completely unstable pelvic ring fractures (type C): a report of 40 cases. J Orthop Trauma 2003;17:555-562. DOI: 10.1097/00005131-200309000-00003.

41. Keel MJ, Benneker LM, et al. Less invasive lumbopelvic stabilization of posterior pelvic ring instability: technique and preliminary results. J Trauma 2011;71:E62-E70. DOI: 10.1097/TA.0b013e3182092e66.

42. Kobbe $\mathrm{P}, \mathrm{Hockertz} \mathrm{I}$, et al. Minimally invasive stabilisation of posterior pelvic-ring instabilities with a transiliac locked compression plate. Int Orthop 2012;36:159-164. DOI: 10.1007/s00264-011-1279-6.

43. Korovessis $P$, Baikousis $A$, et al. Medium- and long-term results of open reduction and internal fixation for unstable pelvic ring fractures. Orthopedics 2000;23:1165-1171.

44. Korovessis $\mathrm{P}$, Stamatakis $\mathrm{M}$, et al. Posterior stabilization of unstable sacroiliac injuries with the Texas Scottish Rite Hospital spinal instrumentation. Orthopedics 2000;23:323-327.

45. Krappinger $D$, Larndorfer $R$, et al. Minimally invasive transiliac plate osteosynthesis for type $C$ injuries of the pelvic ring: a clinical and radiological follow-up. J Orthop Trauma 2007;21:595-602. DOI: 10.1097/BOT.0b013e318158abcf.

46. Laflamme GY, Delisle J, et al. Lateral compression fractures of the superior pubic ramus with intra-articular extension. J Bone Joint Surg Br 2009;91:1208-1212. DOI: 10.1302/0301-620X.91B9.22222.

47. Lindahl J, Hirvensalo E. Outcome of operatively treated type-C injuries of the pelvic ring. Acta Orthop 2005;76:667-678. DOI: 10.1080/17453670510041754.

48. Lindahl J, Makinen TJ, et al. Factors associated with outcome of spinopelvic dissociation treated with lumbopelvic fixation. Injury 2014;45:1914-1920. DOI: 10.1016/j.injury.2014.09.003.

49. Mardanpour K, Rahbar M. The outcome of surgically treated traumatic unstable pelvic fractures by open reduction and internal fixation. J Inj Violence Res 2013;5:77-83. DOI: 10.5249/jivr.v5i2.138.

50. Mason WT, Khan SN, et al. Complications of temporary and definitive external fixation of pelvic ring injuries. Injury 2005;36:599-604. DOI: 10.1016/j.injury.2004.11.016.

51. Matta JM.. Indications for anterior fixation of pelvic fractures. Clin Orthop Relat Res 1996;88-96. DOI: 10.1097/00003086-19960800000011

52. Milenkovic $S$, Mitkovic M, et al. Surgical treatment of the unstable type C pelvic injury. Acta Chir lugosl 2013;60:53-58. DOI: 10.2298/ ACl1302053M.

53. Moed BR, Fissel BA, et al. Percutaneous transiliac pelvic fracture fixation: cadaver feasibility study and preliminary clinical results. J Trauma 2007;62:357-364. DOI: 10.1097/01.ta.0000224191.08250.97; discussion 64.

54. Moed BR, Geer BL. S2 iliosacral screw fixation for disruptions of the posterior pelvic ring: a report of 49 cases. J Orthop Trauma 2006;20:378-383. DOI: 10.1097/00005131-200607000-00002.

55. Moed BR, Whiting DR. Locked transsacral screw fixation of bilateral injuries of the posterior pelvic ring: initial clinical series. J Orthop Trauma 2010;24:616-621. DOI: 10.1097/BOT.0b013e3181df97eb.

56. Muller FJ, Stosiek W, et al. The anterior subcutaneous internal fixator (ASIF) for unstable pelvic ring fractures: clinical and radiological mid-term results. Int Orthop 2013;37:2239-2245. DOI: 10.1007/s00264013-2032-0.
57. Najibi S, Tannast M, et al. Internal fixation of symphyseal disruption resulting from childbirth. J Orthop Trauma 2010;24:732-739. DOI: 10.1097/BOT.0b013e3181d70259.

58. Nicodemo A, Cuocolo C, et al. Minimally invasive reduction of vertically displaced sacral fracture without use of traction table. J Orthop Trauma 2011;12:49-55. DOI: 10.1007/s10195-011-0132-4.

59. Nork $\mathrm{SE}$, Jones $\mathrm{CB}$, et al. Percutaneous stabilization of $\mathrm{U}$-shaped sacral fractures using iliosacral screws: technique and early results. J Orthop Trauma 2001;15:238-246. DOI: 10.1097/00005131-200105000-00002.

60. O'Flanagan SJ, Fulton G, et al. Operative fixation of unstable pelvic ring injuries in polytrauma patients. Ir J Med Sci 1992;161:39-41. DOI: 10.1007/BF02942078.

61. Oh CW, Kim PT, et al. Anterior plating and percutaneous iliosacral screwing in an unstable pelvic ring injury. J Orthop Sci 2008;13: 107-115. DOI: 10.1007/s00776-007-1201-7.

62. Osterhoff G, Ossendorf C, et al. Posterior screw fixation in rotationally unstable pelvic ring injuries. Injury 2011;42:992-996. DOI: 10.1016/ j.injury.2011.04.005.

63. Osterhoff G, Ossendorf C, et al. Percutaneous iliosacral screw fixation in $\mathrm{S} 1$ and $\mathrm{S} 2$ for posterior pelvic ring injuries: technique and perioperative complications. Arch Orthop Trauma Surg 2011;131: 809-813. DOI: 10.1007/s00402-010-1230-0.

64. Park YS, Baek SW, et al. Management of sacral fractures associated with spinal or pelvic ring injury. J Trauma Acute Care Surg 2012;73:239-242. DOI: 10.1097/TA.0b013e31825a79d2.

65. Pohlemann T, Bosch $U$, et al. The Hannover experience in management of pelvic fractures. Clin Orthop Relat Res 1994;69-80. DOI: 10.1097/00003086-199408000-00010.

66. Porter SE, Graves ML, et al. Operative experience of pelvic fractures in the obese. Obesity surgery 2008;18:702-708. DOI: 10.1007/s11695007-9320-y.

67. Putnis SE, Pearce R, et al. Open reduction and internal fixation of a traumatic diastasis of the pubic symphysis: one-year radiological and functional outcomes. J Bone Joint Surg Br 2011;93:78-84. DOI: 10.1302/0301-620X.93B1.23941.

68. Reuther G, Rohner U, et al. CT-guided screw fixation of vertical sacral fractures in local anaesthesia using a standard CT. RoFo 2014;186:1134-1139. DOI: 10.1055/s-0034-1366605.

69. Rommens PM. Pelvic ring injuries: a challenge for the trauma surgeon. Acta chirurgica Belgica 1996;96:78-84.

70. Rommens PM, Hessmann MH. Staged reconstruction of pelvic ring disruption: differences in morbidity, mortality, radiologic results, and functional outcomes between B1, B2/B3, and C-type lesions. J Orthop Trauma 2002;16:92-98. DOI: 10.1097/00005131-200202000-00004.

71. Rommens PM, Vanderschot PM, et al. Surgical management of pelvic ring disruptions. Indications, techniques and functional results. Der Unfallchirurg 1992;95:455-462.

72. Routt ML Jr., Kregor PJ, et al. Early results of percutaneous iliosacral screws placed with the patient in the supine position. J Orthop Trauma 1995;9:207-214. DOI: 10.1097/00005131-199506000-00005.

73. Routt ML Jr., Simonian PT, et al. The retrograde medullary superior pubic ramus screw for the treatment of anterior pelvic ring disruptions: a new technique. J Orthop Trauma 1995;9:35-44. DOI: 10.1097/00005131-199502000-00006.

74. Routt ML Jr., Simonian PT, et al. Iliosacral screw fixation: early complications of the percutaneous technique. J Orthop Trauma 1997;11:584-589. DOI: 10.1097/00005131-199711000-00007.

75. Rysavy M, Pavelka T, et al. Iliosacral screw fixation of the unstable pelvic ring injuries. Acta Chir Orthop Traumatol Cech 2010;77: 209-214.

76. Sagi HC, Militano U, et al. A comprehensive analysis with minimum 1-year follow-up of vertically unstable transforaminal sacral fractures treated with triangular osteosynthesis. J Orthop Trauma 2009;23: 313-319. DOI: 10.1097/BOT.0b013e3181a32b91, discussion 9-21.

77. Sagi HC, Papp S. Comparative radiographic and clinical outcome of two-hole and multi-hole symphyseal plating. J Orthop Trauma 2008;22:373-378. DOI: 10.1097/BOT.0b013e31817e49ee. 
78. Schildhauer TA, Bellabarba C, et al. Decompression and lumbopelvic fixation for sacral fracture-dislocations with spino-pelvic dissociation. J Orthop Trauma 2006;20:447-457. DOI: 10.1097/00005131200608000-00001.

79. Schweitzer D, Zylberberg A, et al. Closed reduction and iliosacral percutaneous fixation of unstable pelvic ring fractures. Injury 2008;39:869-874. DOI: 10.1016/j.injury.2008.03.024.

80. Shuler TE, Boone DC, et al. Percutaneous iliosacral screw fixation: early treatment for unstable posterior pelvic ring disruptions. J Trauma 1995;38:453-458. DOI: 10.1097/00005373-199503000-00031.

81. Soultanis K, Karaliotas $\mathrm{Gl}$, et al. Lumbopelvic fracture-dislocation combined with unstable pelvic ring injury: one stage stabilisation with spinal instrumentation. Injury 2011;42:1179-1183. DOI: 10.1016/ j.injury.2010.06.002.

82. Starr AJ, Nakatani T, et al. Superior pubic ramus fractures fixed with percutaneous screws: what predicts fixation failure? J Orthop Trauma 2008;22:81-87. DOI: 10.1097/BOT.0b013e318162ab6e.

83. Starr AJ, Walter JC, et al. Percutaneous screw fixation of fractures of the iliac wing and fracture-dislocations of the sacro-iliac joint (OTA Types 61-B2.2 and 61-B2.3, or Young-Burgess "lateral compression type II" pelvic fractures). J Orthop Trauma 2002;16:116-123. DOI: 10.1097/00005131-200202000-00008.

84. Stover MD, Sims S, et al. What is the infection rate of the posterior approach to type $C$ pelvic injuries? Clin Orthop Relat Res 2012;470:2142-2147. DOI: 10.1007/s11999-012-2438-9.

85. Suzuki T, Hak DJ, et al. Outcome and complications of posterior transiliac plating for vertically unstable sacral fractures. Injury 2009;40:405-409. DOI: 10.1016/j.injury.2008.06.039.

86. Suzuki $T$, Shindo $M$, et al. Long-term functional outcome after unstable pelvic ring fracture. J Trauma 2007;63:884-888. DOI: 10.1097/01.ta.0000235888.90489.fc.

87. Taguchi $\mathrm{T}$, Kawai $\mathrm{S}$, et al. Operative management of displaced fractures of the sacrum. J Orthop Sci 1999;4:347-352. DOI: 10.1007/ s007760050115.

88. Tan GQ, He JL, et al. Lumbopelvic fixation for multiplanar sacral fractures with spinopelvic instability. Injury 2012;43:1318-1325. DOI: 10.1016/j.injury.2012.05.003.

89. Tee SS, Hyzan Y, et al. Functional outcome of open reduction and internal fixation of pelvic ring injuries. Med J Malaysia 2000;55(Suppl C):49-58.

90. Tornetta P, 3rd, Dickson K. Outcome of rotationally unstable pelvic ring injuries treated operatively. Clin Orthop Relat Res 1996;147-151. DOI: 10.1097/00003086-199608000-00018.

91. Totterman A, Glott T, et al. Unstable sacral fractures: associated injuries and morbidity at 1 year. Spine 2006;31:E628-E635. DOI: 10.1097/01.brs.0000231961.03527.00.

92. Totterman A, Glott T, et al. Pelvic trauma with displaced sacral fractures: functional outcome at one year. Spine 2007;32:1437-1443. DOI: 10.1097/BRS.0b013e318060a68f.

93. Tucker MC, Nork SE, et al. Simple anterior pelvic external fixation. JTrauma 2000;49:989-994. DOI: 10.1097/00005373-200012000-00002.

94. Vaidya $\mathrm{R}$, Colen $\mathrm{R}$, et al. Treatment of unstable pelvic ring injuries with an internal anterior fixator and posterior fixation: initial clinical series. J Orthop Trauma 2012;26:1-8. DOI: 10.1097/BOT.0b013e318233b8a7.

95. Vaidya R, Kubiak EN, et al. Complications of anterior subcutaneous internal fixation for unstable pelvis fractures: a multicenter study. Clin Orthop Relat Res 2012;470:2124-2131. DOI: 10.1007/s11999-0112233-z.

96. Vaidya R, Oliphant BW, et al. Sequential reduction and fixation for windswept pelvic ring injuries (LC3) corrects the deformity until healed. Int Orthop 2013;37:1555-1560. DOI: 10.1007/s00264-013-1891-8.

97. Vallier HA, Cureton BA, et al. Functional outcomes in women after high-energy pelvic ring injury. J Orthop Trauma 2012;26:296-301. DOI: 10.1097/BOT.0b013e318221e94e.

98. Van den Bosch EW, Van der Kleyn R, et al. Functional outcome of internal fixation for pelvic ring fractures. J Trauma 1999;47:365-371. DOI: 10.1097/00005373-199908000-00026.
99. Van Loon P, Kuhn S, et al. Radiological analysis, operative management and functional outcome of open book pelvic lesions: a 13-year cohort study. Injury 2011;42:1012-1019. DOI: 10.1016/j.injury.2010.11.057.

100. van Veen $\mathrm{IH}$, van Leeuwen AA, et al. Unstable pelvic fractures: a retrospective analysis. Injury 1995;26:81-85. DOI: 10.1016/00201383(95)92181-9.

101. Wang $S$, Zhang $P$, et al. Anterior internal fixation to treat vertical unstable pelvic fracture. Chin J Traumatol 2002;5:59-61.

102. Zamzam MM. Unstable pelvic ring injuries. Outcome and timing of surgical treatment by internal fixation. Saudi Med J 2004;25: 1670-1674.

103. Ziran BH, Smith WR, et al. Iliosacral screw fixation of the posterior pelvic ring using local anaesthesia and computerised tomography. J Bone Joint Surg Br 2003;85:411-418. DOI: 10.1302/0301-620X.85B3.13119.

104. Zwingmann J, Sudkamp NP, et al. Intra- and postoperative complications of navigated and conventional techniques in percutaneous iliosacral screw fixation after pelvic fractures: results from the German Pelvic Trauma Registry. Injury 2013;44:1765-1772. DOI: 10.1016/j.injury.2013.08.008.

105. Bi C, Wang Q, et al. Treatment of unstable posterior pelvic ring fracture with pedicle screw-rod fixator vs locking compression plate: a comparative study. Med Sci Monit 2016;22:3764-3770. DOI: 10.12659/ MSM.900673.

106. Elzohairy MM, Salama AM. Open reduction internal fixation vs percutaneous iliosacral screw fixation for unstable posterior pelvic ring disruptions. Orthop Traumatol Surg Res 2017;103(2):223-227.

107. Höch A, Schneider I, et al. Lateral compression type B 2-1 pelvic ring fractures in young patients do not require surgery. Eur J Trauma Emerg Surg 2016;1-7.

108. Hoskins W, Bucknill A, et al. A prospective case series for a minimally invasive internal fixation device for anterior pelvic ring fractures. J Orthop Surg Res 2016;11(1):135. DOI: 10.1186/s13018-016-0468-9.

109. Oh HK, Choo SK, et al. Stoppa approach for anterior plate fixation in unstable pelvic ring injury. Clin Orthop Surg 2016;8:243-248. DOI: 10.4055/cios.2016.8.3.243.

110. Sobhan MR, Abrisham SMJ, et al. Spinopelvic fixation of sacroiliac joint fractures and fracture-dislocations: a clinical 8 years follow-up study. Archives of Bone and Joint Surgery 2016;4:381-386.

111. Leung KS, Chien P, et al. Operative treatment of unstable pelvic fractures. Injury 1992;23:31-37. DOI: 10.1016/0020-1383(92)90122-9.

112. Arazi $M$, Kutlu $A$, et al. The pelvic external fixation: the mid-term results of 41 patients treated with a newly designed fixator. Arch Orthop Trauma Surg 2000;120:584-586. DOI: 10.1007/s004020000142.

113. Chiu FY, Chuang TY, et al. Treatment of unstable pelvic fractures: use of a transiliac sacral rod for posterior lesions and an external fixator for anterior lesions. J Trauma 2004;57:141-144. DOI: 10.1097/01. TA.0000123040.23231.EB.

114. Shirahama M. Surgical treatment of vertically unstable sacral fractures using a new plate. Kurume Med J 2005;52:9-18. DOI: 10.2739/ kurumemedj.52.9.

115. Ayoub MA. Vertically unstable sacral fractures with neurological insult: outcomes of surgical decompression and reconstruction plate internal fixation. Int Orthop 2009;33:261-267. DOI: 10.1007/s00264007-0468-9.

116. Uchida K, Kokubo Y, et al. Fracture of the pelvic ring: a retrospective review of 224 patients treated at a single institution. Eur J Orthop Surg Traumatol 2011;21:251-257. DOI: 10.1007/s00590-010-0706-5.

117. Bates P, Gary J. Percutaneous treatment of pelvic and acetabular fractures in obese patients. Orthop Clin North Am 2011;42:55-67. DOI: $10.1016 /$ j.ocl.2010.08.004.

118. Ayoub MA. Type $C$ pelvic ring injuries in polytrauma patients: can percutaneous iliosacral screws reduce morbidity and costs? Eur J Orthop Surg Traumatol 2012;22:137-144. DOI: 10.1007/s00590011-0811-0.

119. Choy WS, Kim KJ, et al. Anterior Pelvic Plating and Sacroiliac Joint Fixation in Unstable Pelvic Ring Injuries. Yonsei Med J 2012;53: 422-426. DOI: 10.3349/ymj.2012.53.2.422. 
120. Khaled SA, Soliman O, et al. Functional outcome of unstable pelvic ring injuries after iliosacral screw fixation: single vs two screw fixation. Eur J Trauma Emerg Surg 2014;41:387-392. DOI: 10.1007/s00068-0140456-x.

121. Lybrand K, Kurylo J, et al. Does removal of the symphyseal cartilage in symphyseal dislocations have any effect on final alignment and implant failure? J Orthop Trauma 2015;29:470-474. DOI: 10.1097/ BOT.0000000000000376.

122. Sullivan MP, Scolaro JA, et al. Isolated pelvic ring injuries: functional outcomes following percutaneous, posterior fixation. Eur J Orthop Surg Traumatol 2015;25:1025-1030. DOI: 10.1007/s00590-015-1631-4.

123. Zhu $L$, Wang $L$, et al. Treatment of pelvic fractures through a less invasive ilioinguinal approach combined with a minimally invasive posterior approach. BMC Musculoskelet Disord 2015;16:167. DOI: 10.1186/s12891-015-0635-x.

124. Ayoub MA, Gad HM, et al. Standalone percutaneous transiliac plating of vertically unstable sacral fractures: outcomes, complications, and recommendations. Eur Spine J 2016;25:1153-1162. DOI: 10.1007/ s00586-015-3976-0.

125. Hoch A, Schneider I, et al. Lateral compression type B 2-1 pelvic ring fractures in young patients do not require surgery. Eur J Trauma Emerg Surg 2018;44(2):171-177.

126. Lindahl J, Hirvensalo E. Outcome of operatively treated type-C injuries of the pelvic ring. Acta Orthop 2005;76:667-678. DOI: 10.1080/17453670510041754.

127. Moed BR, Whiting DR. Locked transsacral screw fixation of bilateral injuries of the posterior pelvic ring: initial clinical series. J Orthop Trauma 2010;24:616-621. DOI: 10.1097/BOT.0b013e3181df97eb.

128. Putnis $S E$, Pearce $R$, et al. Open reduction and internal fixation of a traumatic diastasis of the pubic symphysis: One-Year Radiological and Functional Outcomes. J Bone Joint Surg Br 2011;93-B:78-84. DOI: 10.1302/0301-620X.93B1.23941.

129. Gänsslen A, Hildebrand F, et al. Supraacetabular external fixation for pain control in geriatric type B pelvic injuries. Acta Chir Orthop Traumatol Cech 2013;80(2):101-105.

130. Gänsslen A, Hüfner T, et al. Percutaneous iliosacral screw fixation of unstable pelvic injuries by conventional fluoroscopy. Oper Orthop Traumatol 2006;18:225-244. DOI: 10.1007/s00064-006-1173-3.

131. Hamad A, Pavlou G, et al. Management of pubic symphysis diastasis with locking plates: a report of 11 cases. Injury 2013;44:947-951. DOI: 10.1016/j.injury.2012.12.018.

132. Osterhoff G, Ossendorf $C$, et al. Posterior screw fixation in rotationally unstable pelvic ring injuries. Injury 2011;42:992-996. DOI: 10.1016/ j.injury.2011.04.005.

133. Bi C, Wang Q, et al. Treatment of Unstable Posterior Pelvic Ring Fracture with Pedicle Screw-Rod Fixator vs Locking Compression Plate: A Comparative Study. Med Sci Monit 2016;22:3764-3770. DOI: 10.12659/MSM.900673.

134. Pennal GF, Tile M, et al. Pelvic disruption: assessment and classification. Clin Orthop Relat Res 1980;12-21. DOI: 10.1097/00003086-19800900000004.

135. Burgess AR, Eastridge $B J$, et al. Pelvic ring disruptions: effective classification system and treatment protocols. J Trauma 1990;30: 848-856. DOI: 10.1097/00005373-199007000-00015.

136. Majeed SA. Grading the outcome of pelvic fractures. J Bone Joint Surg Br 1989;71:304-306. DOI: 10.1302/0301-620X.71B2.2925751.

137. Lindahl J, Hirvensalo $E$, et al. Failure of reduction with an external fixator in the management of injuries of the pelvic ring. Long-term evaluation of 110 patients. J Bone Joint Surg Br 1999;81:955-962.

138. Hontzsch D, Schaser KD, et al. Evaluation of the effectiveness of the angular stable locking system in patients with distal tibial fractures treated with intramedullary nailing: a multicenter randomized controlled trial. J Bone Joint Surg Am 2014;96:1889-1897. DOI: 10.2106/JBJS.M.01355.

139. Thewlis D, Callary SA, et al. Peak loading during walking is not associated with fracture migration following tibial plateau fracture: a preliminary case series. J Orthop Res 2015;33:1398-1406. DOI: 10.1002/ jor.22905.
140. Segal $D$, Mallik AR, et al. Early weight bearing of lateral tibial plateau fractures. Clin Orthop Relat Res 1993;232-237. DOI: 10.1097/00003086199309000-00032.

141. Kenwright J, Richardson JB, et al. Axial movement and tibial fractures. A controlled randomised trial of treatment. J Bone Joint Surg $\mathrm{Br}$ 1991;73:654-659. DOI: 10.1302/0301-620X.73B4.2071654.

142. Helfet DL, Borrelli J Jr., et al. Stabilization of acetabular fractures in elderly patients. J Bone Joint Surg Am 1992;74:753-765. DOI: 10.2106/00004623-199274050-00015.

143. Laflamme GY, Hebert-Davies J, et al. Internal fixation of osteopenic acetabular fractures involving the quadrilateral plate. Injury 2011;42:1130-1134. DOI: 10.1016/j.injury.2010.11.060.

144. Daurka JS, Pastides PS, et al. Acetabular fractures in patients aged $>55$ years: a systematic review of the literature. Bone Joint J 2014 96-B:157-163. DOI: 10.1302/0301-620X.96B2.32979.

145. Rickman M, Young J, et al. Managing acetabular fractures in the elderly with fixation and primary arthroplasty: aiming for early weightbearing. Clin Orthop Relat Res 2014;472:3375-3382. DOI: 10.1007/s11999-014-3467-3.

146. Hutt JR, Ortega-Briones A, et al. The ongoing relevance of acetabular fracture classification. Bone Joint J 2015;97-B:1139-1143. DOI: 10.1302/0301-620X.97B8.33653.

147. The management of hip fracture in adults NICE clinical Guidelines, No 124, National Clinical Guideline Centre (UK), 2011.

148. Rickman M, Young J, et al. The management of complex acetabular fractures in the elderly with fracture fixation and primary total hip replacement. Eur JTrauma Emerg Surg 2012;38:511-516. DOI: 10.1007/ s00068-012-0231-9.

149. Cole RJ, Bindra RR, et al. Radiographic evaluation of osseous displacement following intra-articular fractures of the distal radius: reliability of plain radiography vs computed tomography. J Hand Surg 1997;22:792-800. DOI: 10.1016/S0363-5023(97)80071-8.

150. Mears DC, Velyvis JH. Acute total hip arthroplasty for selected displaced acetabular fractures: two to twelve-year results. The J Bone Joint Surg Am 2002;84-A:1-9. DOI: 10.2106/00004623-200201000-00001.

151. Mouhsine E, Garofalo R, et al. Acute total hip arthroplasty for acetabular fractures in the elderly: 11 patients followed for 2 years. Acta Orthop Scand 2002;73:615-618. DOI: 10.3109/17453670209178024.

152. Herscovici D Jr., Lindvall E, et al. The combined hip procedure: open reduction internal fixation combined with total hip arthroplasty for the management of acetabular fractures in the elderly. J Orthop Trauma 2010;24:291-296. DOI: 10.1097/BOT.0b013e3181b1d22a.

153. Tannast M, Najibi S, et al. Two to twenty-year survivorship of the hip in 810 patients with operatively treated acetabular fractures. J Bone Joint Surg Am 2012;94:1559-1567. DOI: 10.2106/JBJS.K.00444.

154. Moed BR, McMichael JC. Outcomes of posterior wall fractures of the acetabulum. J Bone Joint Surg Am 2007;89:1170-1176. DOI: 10.2106/ JBJS.F.00473.

155. Onsten I, Berzins A, et al. Accuracy and precision of radiostereometric analysis in the measurement of THR femoral component translations: human and canine in vitro models. J Orthop Res 2001;19:1162-1167. DOI: 10.1016/S0736-0266(01)00039-0.

156. Bragdon $\mathrm{CR}$, Malchau $\mathrm{H}$, et al. Experimental assessment of precision and accuracy of radiostereometric analysis for the determination of polyethylene wear in a total hip replacement model. J Orthop Res 2002;20:688-695. DOI: 10.1016/S0736-0266(01)00171-1.

157. Bragdon CR, Estok DM, et al. Comparison of two digital radiostereometric analysis methods in the determination of femoral head penetration in a total hip replacement phantom. J Orthop Res 2004;22:659-664. DOI: 10.1016/j.orthres.2003.09.004.

158. Solomon LB, Callary SA, et al. Weight-bearing-induced displacement and migration over time of fracture fragments following split depression fractures of the lateral tibial plateau: a case series with radiostereometric analysis. J Bone Joint Surg Br 2011;93:817-823. DOI: 10.1302/0301-620X.93B6.26122.

159. Solomon LB, Stevenson AW, et al. The accuracy and precision of radiostereometric analysis in monitoring tibial plateau fractures. Acta Orthop 2010;81:487-494. DOI: 10.3109/17453674.2010.487930. 